\title{
On dynamically consistent Jacobian inverse for non-holonomic robotic systems
}

\author{
JOANNA RATAJCZAK and KRZYSZTOF TCHOŃ
}

\begin{abstract}
This paper presents the dynamically consistent Jacobian inverse for non-holonomic robotic system, and its application to solving the motion planning problem. The system's kinematics are represented by a driftless control system, and defined in terms of its input-output map in accordance with the endogenous configuration space approach. The dynamically consistent Jacobian inverse (DCJI) has been introduced by means of a Riemannian metric in the endogenous configuration space, exploiting the reduced inertia matrix of the system's dynamics. The consistency condition is formulated as the commutativity property of a diagram of maps. Singular configurations of DCJI are studied, and shown to coincide with the kinematic singularities. A parametric form of DCJI is derived, and used for solving example motion planning problems for the trident snake mobile robot. Some advantages in performance of DCJI in comparison to the Jacobian pseudoinverse are discovered.
\end{abstract}

Key words: non-holonomic system, motion, Jacobian inverse, dynamic consistency, metric, motion planning

\section{Introduction}

It is well known that for both holonomic as well as non-holonomic robotic systems the motion planning problem can be solved by means of Jacobian algorithms. A systematic way of derivation of the Jacobian algorithms is offered by the continuation method introduced into robotics by H. Sussmann [20]. Within this paradigm, in [21] we have introduced the endogenous configuration space approach, and derived Jacobian inverses and Jacobian motion planning algorithms for non-holonomic systems. It is worth mentioning that the endogenous configuration space approach allows to transfer the concepts originally defined for holonomic systems to the world of non-holonomic systems, preserving conceptual consistency of both these areas. An up to date review of this approach has been provided recently in [23].

The authors are with Department of Cybernetics and Robotics, Wrocław University of Science and Technology, ul. Janiszewskiego 11/17, 50-372 Wrocław, Poland.

E-mails: \{joanna.ratajczak\}\{krzysztof.tchon\}@ pwr.edu.pl

This research was supported by the Wrocław University of Science and Technology under a statutory research project.

Received 26.01.2017. 
An example of such a transferable concept is the dynamically consistent Jacobian inverse (DCJI) developed for robotic manipulators by O. Khatib [8, 9], and then exploited successfully to the control of redundant manipulators [12], humanoid robots [10, 18] as well as biomechanical systems $[3,4]$. In the original Khatib's formulation the dynamic consistency of a Jacobian inverse means that the forces acting in the configuration space and not transmitted by the dual Jacobian inverse to the operational space do not cause any motion of the end effector. When resolved mathematically, this postulate results in a kind of the weighted version of the Jacobian pseudoinverse [7, 2], where the role of the weight matrix is performed by the inertia matrix of the manipulator. In this way DCJI becomes a tool for inversion of the kinematics, respecting some features of the manipulator's dynamics.

Following the idea of DCJI for holonomic robotic systems, in [22] we have designed DCJI for non-holonomic robotic systems. Three methods of the derivation have been promoted in this reference, called the geometric, the force, and the optimization method; the force method refers directly to the Khatib's approach. DCJI for mobile manipulators is devised in [17].

The purpose of this paper is to present and further advance the geometric method of designing DCJI for non-holonomic robotic systems. We claim that DCJI originates from a sort the interplay among a number of maps characterizing the infinitesimal behaviour of the robotic system including the Jacobian, the Jacobian inverse, and their dual maps. In accordance with [22], within the geometric method a leading actor is the Riemannian metric in the endogenous configuration space defined by means of the reduced inertia matrix of the system's dynamics, and the related isomorphisms between the endogenous configuration space and its dual space. All these maps have been arranged into a diagram whose commutativity guarantees the dynamic consistency of the Jacobian inverse. Two aspects of this commutativity are considered, both leading to the same formula for DCJI. Geometrically, the dynamic consistency prevents the motion of the end effector under the influence of the endogenous momenta contained in the null space of the dual Jacobian inverse. Associated with DCJI is the motion planning algorithm allowing to compute the control function that makes the end effector to reach a desired point in the operational space. This algorithm is based on solving a functional differential equation evolving in the endogenous configuration space. Therefore, in order to facilitate the computation of its solution the control functions have been replaced by their truncated orthogonal series, making the motion planning algorithm finite dimensional. In the paper a parametric form of all the concepts underlying the derivation of DCJI is provided, resulting in a parametric dynamically consistent Jacobian motion algorithm. Performance of this algorithm is illustrated with a motion planning problem for two kinematics models of the trident snake robot $[5,6]$, differing from each other by the choice of control inputs. A comparison with the motion planning algorithm based on the classical Jacobian pseudoinverse reveals some advantages of DCJI.

This paper is organized in the following way. The next section introduces basic concepts, including the kinematics, the endogenous configuration, the Jacobian, the Jacobian inverse, configuration singularities, and the dynamics model of a non-holonomic system. 
Section 3 is devoted to DCJI, that is derived on the basis of a Riemannian metric, from the commutativity requirement of a diagram of maps. To aid the computations, in section 4 a parametric version of the concepts introduced in the previous section is defined, including the diagram of parametric maps and a parametric dynamically consistent Jacobian motion planning algorithm. Example computations are conducted in section 5 in relation to a motion planning problem for two kinematics models of the trident snake robot. Section 6 concludes the paper.

\section{Basic concepts}

We shall study robotic system characterized by configuration coordinates $q \in Q=\mathbf{R}^{n}$ and velocities $\dot{q} \in T_{q} Q$, subject to $l \leqslant n$ independent constraints in the form of Pfaff

$$
\mathrm{A}(q) \dot{q}=0 .
$$

Hereabout $T_{q} Q \cong \mathbf{R}^{n}$ denotes the tangent space to $Q$ at the configuration $q$, and the matrix $\mathrm{A}(q)$ has dimension $l \times n$ and full rank $l$. It will be assumed that the constraints (1) are non-holonomic.

After adding an output function, the Pfaffian constraints provide a description of the system's kinematics in the form of a driftless control system with output

$$
\dot{q}=G(q) u=\sum_{i=1}^{m} g_{i}(q) u_{i}, \quad y=k(q)=\left(k_{1}(q), \ldots, k_{r}(q)\right),
$$

whose vector fields $g_{i}(q)$ belong to the null space of the Pfaffian matrix $\mathrm{A}(q)$. The control vector $u \in \mathbf{R}^{m}, m=n-l$, and the output vector $y \in Y=\mathbf{R}^{r}$ comprises operational coordinates. It will be assumed that the controls act on the system (2) over a time interval $[0, T]$, and its control functions $u(\cdot)$ belong to the Hilbert space $X=L_{m}^{2}[0, T]$ of Lebesgue square integrable functions of time, with values in w $\mathbf{R}^{m}$. The control space $\mathcal{X}$ is equipped with the inner product

$$
\left\langle u_{1}(\cdot), u_{2}(\cdot)\right\rangle=\int_{0}^{T} u_{1}^{T}(t) u_{2}(t) d t .
$$

In accordance with the terminology adopted in [21, 23], this space will be referred to as the endogenous configuration space of the system (2). Concerning the control functions of (2) our standing assumption will be that for every $u(\cdot) \in \mathcal{X}$ and every initial configuration $q_{0}$ the system's trajectory $q(t)=\varphi_{q_{0}, t}(u(\cdot))$ exists for every $t \in[0, T]$.

In agreement with the endogenous configuration space approach, the kinematics of the robotic system will be identified with the input-output map

$$
K_{q_{0}, T}: X \longrightarrow Y
$$


of (2), defined as

$$
K_{q_{0}, T}(u(\cdot))=k(q(T))=k\left(\varphi_{q_{0}, T}(u(\cdot))\right) .
$$

The map (5) is continuously differentiable, see [11], Appendix D. Its derivative with respect to the control function,

$$
J_{q_{0}, T}(u(\cdot)) v(\cdot)=D K_{q_{0}, T}(u(\cdot)) v(\cdot)=\left.\frac{d}{d \alpha}\right|_{\alpha=0} K_{q_{0}, T}(u(\cdot)+\alpha v(\cdot)),
$$

will be regarded as the Jacobian of the non-holonomic robotic system. At a fixed control function $u(\cdot)$ the Jacobian

$$
J_{q_{0}, T}(u(\cdot)): T_{u(\cdot)} X \longrightarrow T_{K_{q_{0}, T}(u(\cdot))} Y
$$

becomes a linear map between corresponding tangent spaces $T_{u(\cdot)} X \cong X$ and $T_{K_{q_{0}, T}(u(\cdot))} Y \cong Y$. It is well known [19], that for a given $(u(t), q(t))$ the Jacobian is determined by the linear approximation of the system (2), so setting $\xi(t)=D \varphi_{q_{0}, t}(u(\cdot)) v(\cdot)$, $v \in \mathbf{R}^{m}$ and $w \in \mathbf{R}^{r}$, we get a linear control system with output

$$
\dot{\xi}=A(t) \xi+B(t) v, \quad w=C(t) \xi
$$

where $\xi_{0}=0$ and

$$
A(t)=\frac{\partial G(q(t)) u(t)}{\partial q}, \quad B(t)=G(q(t)), \quad C(t)=\frac{\partial k(q(t))}{\partial q} .
$$

Having solved the system (8), we obtain the following integral formula for the Jacobian

$$
J_{q_{0}, T}(u(\cdot)) v(\cdot)=w(T)=C(T) \xi(T)=C(T) \int_{0}^{T} \Phi(T, t) B(t) v(t) d t .
$$

The matrix $\Phi(t, s)$ is the fundamental matrix of system (8), satisfying the differential equation

$$
\frac{\partial \Phi(t, s)}{\partial t}=A(t) \Phi(t, s), \quad \Phi(s, s)=I_{n}
$$

The Jacobian (7) has its dual

$$
J_{q_{0}, T}^{*}(u(\cdot)): \mathbf{R}^{r *} \longrightarrow X^{*}
$$

acting between suitable dual (cotangent) spaces. For $r^{T} \in \mathbf{R}^{r *}$ and $v(\cdot) \in X$ the dual (12) is defined as follows

$$
\left(J_{q_{0}, T}^{*}(u(\cdot)) r^{T}\right) v(\cdot)=r^{T} J_{q_{0}, T}(u(\cdot)) v(\cdot)=r^{T} C(T) \int_{0}^{T} \Phi(T, t) B(t) v(t) d t .
$$


In particular, (13) implies that the dual Jacobian is determined by the matrix function

$$
\bar{J}_{q_{0}, T}(u(\cdot))(t)=B^{T}(t) \Phi^{T}(T, t) C^{T}(T),
$$

such that, using the inner product (3), we obtain

$$
\left(J_{q_{0}, T}^{*}(u(\cdot)) r^{T}\right) v(\cdot)=\left\langle\bar{J}_{q_{0}, T}(u(\cdot))(\cdot) r, v(\cdot)\right\rangle .
$$

Relying on this identity, and exploiting the identifications $X^{*} \cong X$ and $R^{r *} \cong R^{r}$ we derive the identity

$$
\begin{gathered}
J_{q_{0}, T}(u(\cdot)) J_{q_{0}, T}^{*}(u(\cdot))=C(T) \int_{0}^{T} \Phi(T, t) B(t) B^{T}(t) \Phi^{T}(T, t) d t= \\
C(T) G(T) C^{T}(T)=\mathcal{G}_{q_{0}, T}(u(\cdot)) .
\end{gathered}
$$

The matrix $\mathcal{G}_{q_{0}, T}(u(\cdot))$ standing in (15) will be referred to as the mobility matrix of the non-holonomic robotic system.

Having defined the kinematics of the non-holonomic robotic system as the map (4), by reference to the Jacobian (7) we can introduce regular and singular endogenous configurations, [21]. Specifically, an endogenous configuration $u(\cdot) \in \mathcal{X}$, at which the Jacobian is surjective is called regular, otherwise $u(\cdot)$ is singular. It follows that singular configurations coincide with singular optimal controls of the system (2). Equivalently, $u(\cdot) \in X$ is singular if and only if at $u(\cdot)$ the mobility matrix (15) loses rank. It is easily checked that the matrix $G(t)=\int_{0}^{t} \Phi(t, s) B(s) B^{T}(s) \Phi^{T}(t, s) d s$ constituting the mobility matrix satisfies the Lyapunov equation

$$
\dot{G}(t)=B(t) B^{T}(t)+A(t) G(t)+G(t) A^{T}(t), \quad G(0)=0 .
$$

By definition, at a regular endogenous configuration the Jacobian equation

$$
J_{q_{0}, T}(u(\cdot)) v(\cdot)=w
$$

delivers a solution $v(\cdot) \in X$ for any $w \in R^{r}$. Such a solution can be found by means of a right Jacobian inverse

$$
J_{q_{0}, T}^{\#}(u(\cdot)): T_{K_{q_{0}, T}(u(\cdot))} Y \longrightarrow T_{u(\cdot)} X,
$$

that satisfies the identity $J_{q_{0}, T}(u(\cdot)) J_{q_{0}, T}^{\#}(u(\cdot))=I_{r}$. A well known example of the right inverse is the Jacobian pseudoinverse (the Moore-Penrose generalized Jacobian inverse, [1])

$$
J_{q_{0}, T}^{\# M P}(u(\cdot))(t)=B^{T}(t) \Phi^{T}(T, t) C^{T}(T) \mathcal{G}_{q_{0}, T}^{-1}(u(\cdot)),
$$

defined as the solution of a minimal quadratic optimal control problem in the system (8), satisfying the equality condition (17), [21]. Right Jacobian inverses can be employed in order to solve the inverse kinematics (so the motion planning problem) in 
the non-holonomic robotic system. The problem consists in computing a right inverse $L_{q_{0}, T}: Y \longrightarrow X$ of the kinematics, such that $K_{q_{0}, T} \circ L_{q_{0}, T}=i d_{Y}$. This objective can be achieved by applying the continuation method, in agreement with the procedure sketched below. We begin with an initial guess $u_{0}(\cdot) \in X$. If it solves the problem, we finish. Otherwise, we look for a continuously differentiable curve $u_{\theta}(\cdot)$ of endogenous configurations, such that the error $e(\theta)=K_{q_{0}, T}\left(u_{\theta}(\cdot)\right)-y_{d}$ decays exponentially to zero, with a pre-defined decay rate $\gamma>0$. This requirement will be fulfilled, if the curve $u_{\theta}(\cdot)$ satisfies the differential equation

$$
e^{\prime}(\theta)=\frac{d e(\theta)}{d \theta}=-\gamma e(\theta) .
$$

Now, taking into account the form of the error, we arrive at an implicit differential equation

$$
J_{q_{0}, T}\left(u_{\theta}(\cdot)\right) u_{\theta}^{\prime}(\cdot)=-\gamma e(\theta) .
$$

Finally, having applied a right inverse $J^{\#}(u(\cdot))$ of the Jacobian, we convert this equation to the explicit form

$$
u_{\theta}^{\prime}(\cdot)=-\gamma J^{\#}\left(u_{\theta}(\cdot)\right)\left(K_{q_{0}, T}\left(u_{\theta}(\cdot)\right)-y_{d}\right), \quad u_{\theta=0}(\cdot)=u_{0}(\cdot) .
$$

The differential equation (20) establishes a Jacobian inverse kinematics algorithm. Let $u_{\theta}(\cdot)\left(y_{d}\right)$ denote its solution corresponding to $y_{d}$. Then, the solution of the inverse kinematics problem is obtained as the limit

$$
u(t)\left(y_{d}\right)=\lim _{\theta \longrightarrow+\infty} u_{\theta}(t)\left(y_{d}\right),
$$

In this setting, the value $L_{q_{0}, T}\left(y_{d}\right)$ of the inverse kinematics can be computed as the limit

$$
L_{q_{0}, T}\left(y_{d}\right)=\lim _{\theta \longrightarrow+\infty} u_{\theta}(\cdot)\left(y_{d}\right) .
$$

The last issue examined in this section is concerned with the dynamics of the nonholonomic system. Given the Pfaffian constraints (1) or the control system (2), we define the Lagrangian $L(q, \dot{q})=\frac{1}{2} \dot{q}^{T} M(q) \dot{q}-V(q)$, where $M(q)=M^{T}(q)>0$ is the inertia matrix and $V(q)$ denotes the potential energy. Then, the Euler-Lagrange equations of motion take the form

$$
M(q) \ddot{q}+N(\dot{q}, q)=B(q) \tau+T,
$$

where $N(\dot{q}, q)$ represents the centripetal, Coriolis and gravity terms, $B(q)$ is the control matrix, $\tau$ stands for driving forces or torques, and $T$ denotes the traction forces enforcing the satisfaction of motion constraints. Having applied the d'Alembert Principle we compute the traction forces, and then eliminate them that results in the reduced equations of motion

$$
\dot{q}=G(q) u, \quad F(q) \dot{u}+P(q, u)=S(q) \tau .
$$

For further use we pay special attention to the reduced inertia matrix

$$
F(q)=G^{T}(q) M(q) G(q) .
$$




\section{Dynamically consistent Jacobian inverse}

Consider the non-holonomic robotic system with the kinematics (2), the Jacobian (10), and the reduced inertia matrix (24). Suppose that $J_{q_{0}, T}^{\#}(u(\cdot))$ is a right inverse (18) of the Jacobian. Given this right inverse, the map $L_{q_{0}, T}(y)$ can be computed by the related Jacobian inverse kinematics algorithm, in accordance with (20) and (22).

In this section we shall describe a specific inverse, called the Dynamically Consistent Jacobian Inverse (DCJI). In the reference [22] we presented three methods of derivation of DCJI. The reconstruction presented here relies on the method referred to as geometric. As a preliminary step we endow the endogenous configuration space with a Riemannian metric based on the matrix (24). Given an endogenous configuration $u(\cdot) \in \mathcal{X}$, we let $q(t)=\varphi_{q_{0}, t}(u(\cdot))$ denote the corresponding trajectory of (2). Set

$$
\mathcal{M}_{q_{0}}(u(\cdot))(t)=F\left(\varphi_{q_{0}, t}(u(\cdot))\right),
$$

and choose a pair of elements $v_{1}(\cdot), v_{2}(\cdot) \in T_{u(\cdot)} X$. Then, the Riemannian metric on $X$ can be defined in the following way

$$
g_{X}(u(\cdot))\left(v_{1}(\cdot), v_{2}(\cdot)\right)=\int_{0}^{T} v_{1}^{T}(t) \mathcal{M}_{q_{0}}(u(\cdot))(t) v_{2}(t) d t .
$$

Associated with (25) are two isomorphisms

$$
g_{X}^{b}(u(\cdot)): T_{u(\cdot)} X \longrightarrow T_{u(\cdot)}^{*} X \quad \text { and } \quad g_{X}^{\sharp}(u(\cdot)): T_{u(\cdot)}^{*} X \longrightarrow T_{u(\cdot)} X,
$$

often called musical, defined as

$$
\begin{aligned}
& g_{X}^{b}(u(\cdot))(v(\cdot))=v^{T}(\cdot) \mathcal{M}_{q_{0}}(u(\cdot))(\cdot)=p^{T}(\cdot), \\
& g_{X}^{\sharp}(u(\cdot))\left(p^{T}(\cdot)\right)=\mathcal{M}_{q_{0}}^{-1}(u(\cdot))(\cdot) p(\cdot)=v(\cdot),
\end{aligned}
$$

where $v(\cdot) \in T_{u(\cdot)} X$ and $p^{T}(\cdot) \in T_{u(\cdot)}^{*} X$ play the role of the endogenous velocity and the endogenous momentum at the configuration $u(\cdot)$. Observe that the identities in (26) should be read as

$$
p^{T}(t)=v^{T}(t) \mathcal{M}_{q_{0}}(u(\cdot))(t) \quad \text { and } \quad v(t)=\mathcal{M}_{q_{0}}^{-1}(u(\cdot))(t) p(t) .
$$

By definition, the isomorphisms (26) are mutually inverse, so

$$
g_{X}^{\sharp}(u(\cdot)) \circ g_{X}^{b}(u(\cdot))=i d_{T_{u(\cdot)}} x .
$$

Moreover, the action of $p^{T}(\cdot)$ on $v(\cdot)$ can be interpreted as energy, and is tantamount to the usual pairing

$$
p^{T}(\cdot) v(\cdot)=\langle p(\cdot), v(\cdot)\rangle=\int_{0}^{T} p^{T}(t) v(t) d t,
$$


$\langle\cdot, \cdot\rangle$ denoting the inner product (3) in the endogenous configuration space. The operational space is also equipped with a Riemannian metric $g_{Y}(y)$, that will be specified later. The musical isomorphisms associated with this metric are denoted as $g_{Y}^{b}(y)$ and $g_{Y}^{\sharp}(y)$.

Now we are ready to introduce DCJI, following the geometric method. Given the Jacobian (10) and its right inverse (18), we take the dual maps

$$
J_{q_{0}, T}^{*}(u(\cdot)): T_{y}^{*} Y \longrightarrow T_{u(\cdot)}^{*} X \quad \text { and } \quad J_{q_{0}, T}^{\# *}(u(\cdot)): T_{u(\cdot)}^{*} X \longrightarrow T_{y}^{*} Y
$$

operating as

$$
\begin{aligned}
\left(J_{q_{0}, T}^{*}(u(\cdot)) r^{T}\right) v(\cdot)=r^{T} J_{q_{0}, T}(u(\cdot)) v(\cdot) \quad \text { and } & \\
& \left(J_{q_{0}, T}^{\# *}(u(\cdot)) p^{T}(\cdot)\right) w=p^{T}(\cdot) J_{q_{0}, T}^{\#}(u(\cdot)) w .
\end{aligned}
$$

The maps introduced in sections 2 and 3 can be arranged into the following diagram

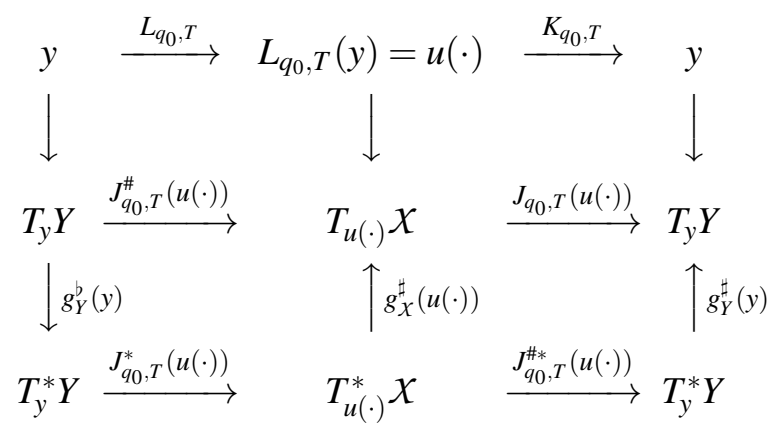

The right Jacobian inverse $J_{q_{0}, T}^{\#}(u(\cdot))$ is dynamically consistent, if the lower subdiagram of (29) commutes. The resulting DCJI will be denoted by $J_{q_{0}, T}^{\# D C}(u(\cdot))$. The diagram's commutativity has two aspects. If the lower left subdiagram commutes then, for $y=$ $K_{q_{0}, T}(u(\cdot))$, we get

$$
J_{q_{0}, T}^{\# D C}(u(\cdot))=g_{X}^{\sharp}(u(\cdot)) J_{q_{0}, T}^{*}(u(\cdot)) g_{Y}^{b}(y) .
$$

A composition from the left with $J_{q_{0}, T}(u(\cdot))$ results in

$$
g_{Y}^{b}(y)=\left(J_{q_{0}, T}(u(\cdot)) g_{X}^{\sharp}(u(\cdot)) J_{q_{0}, T}^{*}(u(\cdot))\right)^{-1},
$$

where $u(\cdot)=L_{q_{0}, T}(y)$. Finally, using (26), we conclude that

$$
J_{q_{0}, T}^{\# D C}(u(\cdot))=g_{X}^{\sharp}(u(\cdot)) J_{q_{0}, T}^{*}(u(\cdot))\left(J_{q_{0}, T}(u(\cdot)) g_{X}^{\sharp}(u(\cdot)) J_{q_{0}, T}^{*}(u(\cdot))\right)^{-1} .
$$

Re-stated in matrix terms, for $w \in T_{y} Y$, the identity (30) is equivalent to

$$
\left(J_{q_{0}, T}^{\# D C}(u(\cdot)) w\right)(t)=\mathcal{M}_{q_{0}}^{-1}(u(\cdot))(t) B^{T}(t) \Phi^{T}(T, t) C^{T}(T)\left(\mathcal{D}_{q_{0}, T}^{D C}\right)^{-1}(u(\cdot)) w .
$$


The matrix

$$
\mathcal{D}_{q_{0}, T}^{D C}(u(\cdot))=C(T) \int_{0}^{T} \Phi(T, t) B(t) \mathcal{M}_{q_{0}}^{-1}(u(\cdot))(t) B^{T}(t) \Phi^{T}(T, t) d t C^{T}(T)
$$

appearing in (31) is called the dynamically consistent mobility matrix. By analogy with (15), we set

$$
\mathcal{D}_{q_{0}, T}^{D C}(u(\cdot))=C(T) D(T) C^{T}(T),
$$

and derive the Lyapunov equation

$$
\dot{D}(t)=B(t) \mathcal{M}_{q_{0}}^{-1}(u(\cdot))(t) B^{T}(t)+A(t) D(t)+D(t) A^{T}(t), \quad D(0)=0 .
$$

Thanks to the positive definiteness of the matrix (24), the singular endogenous configurations of mobility matrices (15) and (32) coincide. As a by-product of the mathematical developments we have obtained the Riemannian metric in the operational space that should be defined as

$$
g_{Y}(y)\left(w_{1}, w_{2}\right)=w_{1}^{T}\left(\mathcal{D}_{q_{0}, T}^{D C}\right)^{-1}\left(L_{q_{0}, T}(y)\right) w_{2},
$$

Even more transparently the dynamic consistency is visible from the analysis of the lower right subdiagram of (29). This is so because the dynamic consistency requires that endogenous momenta belonging to the null space of $J_{q_{0}, T}^{\# *}(u(\cdot))$, so annihilated by this map, should never produce any velocity of motion in the operational space $Y$. In mathematical terms

$$
\operatorname{ker} J_{q_{0}, T}^{\# D C *}(u(\cdot)) \subset \operatorname{ker} J_{q_{0}, T}(u(\cdot)) g_{X}^{\sharp}(u(\cdot)) .
$$

Now, since the null space of any dual right Jacobian inverse $J_{q_{0}, T}^{\# *}(u(\cdot))$ is spanned by

$$
i d_{T_{u(\cdot)}^{*}}^{*} x-J_{q_{0}, T}^{*}(u(\cdot)) J_{q_{0}, T}^{\# *}(u(\cdot)),
$$

the identity (36) results in

$$
J_{q_{0}, T}(u(\cdot)) g_{X}^{\sharp}(u(\cdot))\left(i d_{T_{u(\cdot)}^{*}} x-J_{q_{0}, T}^{*}(u(\cdot)) J_{q_{0}, T}^{\# D C *}(u(\cdot))\right)=0,
$$

from which (30) follows directly.

\section{Parametric DCJI}

Since the DCJI relies on the functional differential equation (20) evolving on the infinite dimensional endogenous configuration space, for computational purposes it may 
be advantageous to introduce a finite dimensional parametrization of endogenous configurations (control functions). This is realized by some truncated orthogonal series exploiting a finite number of basic functions $f_{1}(t), f_{2}(t), \ldots, f_{k}(t)$. To this aim, suppose that the matrix

$$
P(t)=\operatorname{blockdiag}\left\{P_{1}(t), P_{2}(t), \ldots, P_{m}(t)\right\}, \quad P_{i}(t)=\left[f_{1}(t), f_{2}(t), \ldots, f_{k}(t)\right],
$$

of dimension $m \times s, s=m k$ collects these basic functions, suitably arranged, so that

$$
u(t)=u_{\lambda}(t)=P(t) \lambda, \quad \lambda=\left(\lambda_{1}, \lambda_{2}, \ldots, \lambda_{s}\right) \in \mathbf{R}^{s} .
$$

The orthogonality condition means that $\int_{0}^{T} P^{T}(t) P(t) d t=I_{s}$. This parametrization is $s$ dimensional, with $s$ arbitrary, but not less than the dimension of the operational space, $s \geqslant r$. After replacing in (2) and in the following equations the control function by the parametric form (38) we obtain the parametric trajectory

$$
\widetilde{\varphi}_{q_{0}, t}(\lambda)=\varphi_{q_{0}, t}\left(u_{\lambda}(\cdot)\right)
$$

and the parametric kinematics

$$
\widetilde{K}_{q_{0}, T}(\lambda)=K_{q_{0}, T}\left(u_{\lambda}(\cdot)\right) .
$$

Next, along the pair $\left(u_{\lambda}(t), \widetilde{\varphi}_{q_{0}, t}(\lambda)\right)$ we compute the parametric forms $A_{\lambda}(t), B_{\lambda}(t)$, $C_{\lambda}(t)$ of matrices (9) then $\Phi_{\lambda}(t, s)$, arriving at the parametric Jacobian

$$
\widetilde{J}_{q_{0}, T}(\lambda)=C_{\lambda}(T) \int_{0}^{T} \Phi_{\lambda}(T, t) B_{\lambda}(t) P(t) d t,
$$

and the parametric inertia matrix

$$
\widetilde{\mathcal{M}}_{q_{0}}(\lambda)(t)=F\left(\widetilde{\varphi}_{q_{0}, t}(\lambda)\right) .
$$

These data allow one to compute the parametric Riemannian metric

$$
\widetilde{g}_{\mathbf{R}^{s}}(\lambda)\left(\mu_{1}, \mu_{2}\right)=\mu_{1}^{T} \mathcal{R}_{q_{0}, T}(\lambda) \mu_{2},
$$

where a pair of parametric endogenous $\mu_{1}, \mu_{2} \in \mathbf{R}^{s}$, based on the matrix

$$
\mathcal{R}_{q_{0}, T}(\lambda)=\int_{0}^{T} P^{T}(t) \widetilde{\mathcal{M}}_{q_{0}, T}(\lambda)(t) P(t) d t .
$$

In consequence, the musical isomorphisms (26) take the following parametric form

$$
\widetilde{g}_{\mathbf{R}^{s}}^{b}(\lambda)(\mu)=\mu^{T} \mathcal{R}_{q_{0}, T}(\lambda)=p^{T} \quad \text { and } \quad \widetilde{g}_{\mathbf{R}^{s}}^{\sharp}(\lambda)(p)=\mathcal{R}_{q_{0}, T}^{-1}(\lambda) p=\mu,
$$


where $p^{T} \in \mathbf{R}^{s *} \cong \mathbf{R}^{s}$ denotes the parametric endogenous momentum.

Having defined the parametric Jacobian, we introduce the parametric right Jacobian

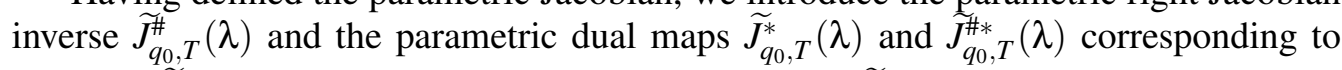
(28). Let $\widetilde{L}_{q_{0}, T}(\lambda)$ stand for the parametric inverse of $\widetilde{K}_{q_{0}, T}(\lambda)$. Then, analogically to (29), for a fixed $\lambda$ all these parametric maps will be embedded into the diagram of maps

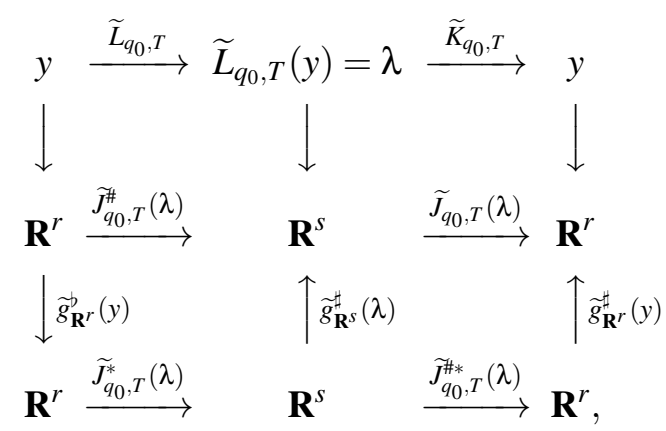

where we have used the obvious identifications $\mathbf{R}^{s *} \cong \mathbf{R}^{s}$ and $\mathbf{R}^{r *} \cong \mathbf{R}^{r}$.

Now, assuming commutativity of the lower left subdiagram of (39), we deduce that for DCJI

$$
\widetilde{J}_{q_{0}, T}^{\# D C}(\lambda)=\widetilde{g}_{\mathbf{R}^{s}}^{\sharp}(\lambda) \widetilde{J}_{q_{0}, T}^{*}(\lambda) \widetilde{g}_{\mathbf{R}^{r}}^{\phi}(y), \quad y=\widetilde{K}_{q_{0}, T}(\lambda) .
$$

After some further developments resembling those preceding (31) we reach a conclusion that the parametric DCJI takes the following form

$$
\widetilde{J}_{q_{0}, T}^{\# D C}(\lambda)=\mathcal{R}_{q_{0}, T}^{-1}(\lambda) \widetilde{J}_{q_{0}, T}^{T}(\lambda)\left(\widetilde{\mathcal{D}}_{q_{0}, T}^{D C}(\lambda)\right)^{-1}
$$

where

$$
\widetilde{\mathcal{D}}_{q_{0}, T}^{D C}(\lambda)=\widetilde{J}_{q_{0}, T}(\lambda) \mathcal{R}_{q_{0}, T}^{-1}(\lambda) \widetilde{J}_{q_{0}, T}^{T}(\lambda)
$$

stands for a parametric dynamically consistent mobility matrix.

To comply with the request that the parametric momenta living in the null space of $\widetilde{J}_{q_{0}, T}^{* *}(\lambda)$ should not affect the motion in the operational space, the lower right subdiagram of (39) yields

$$
\operatorname{ker} \widetilde{J}_{q_{0}, T}^{\# D C *}(\lambda) \subset \operatorname{ker} \widetilde{J}_{q_{0}, T}(\lambda) g_{\mathbf{R}^{s}}^{\sharp}(\lambda),
$$

so adopting a reasoning analogous to that after (36) we come again to the identity (40).

In order to introduce the parametric dynamically consistent Jacobian motion planning algorithm we take a continuously differentiable curve $\lambda_{\theta} \in \mathbf{R}^{s}$ and the corresponding parametric curve of controls $u_{\theta}(t)=P(t) \lambda_{\theta}$ in the endogenous configuration space $x$. Let

$$
\widetilde{e}_{\theta}=\widetilde{K}_{q_{0}, T}\left(\lambda_{\theta}\right)-y_{d}
$$

denote a parametric error curve in the operational space. The parametric dynamically consistent Jacobian motion planning algorithm is determined by the trajectory $\lambda_{\theta}$ of the 
following differential equation resulting from the substitution of (40) into (20)

$$
\lambda_{\theta}^{\prime}=-\gamma \mathcal{R}_{q_{0}, T}^{-1}\left(\lambda_{\theta}\right) \widetilde{J}_{q_{0}, T}^{T}\left(\lambda_{\theta}\right)\left(\widetilde{\mathcal{D}}_{q_{0}, T}^{D C}\left(\lambda_{\theta}\right)\right)^{-1}\left(\widetilde{K}_{q_{0}, T}\left(\lambda_{\theta}\right)-y_{d}\right) .
$$

In the next section the algorithm defined by (41) will solve example motion planning problem for the trident snake robot.

\section{Computations}

The trident snake robot, depicted in Fig. 1, is composed of a triangular shape body and three movable links fixed to the body and at the vertexes of the triangle by revolute joints. Each link is supported by a passive wheel moving without the lateral slip. The trident snake has been originally introduced in [5] as an example of a non-holonomic system with 3 controls and the bracket generating property achieved by the control vector fields and their first order Lie brackets. It also serves as an example of a robotic system with so called undulatory locomotion [13]. The study of kinematics of the trident snake was done in $[5,6]$, while the trident snake with dynamics was examined in $[15,16]$. The

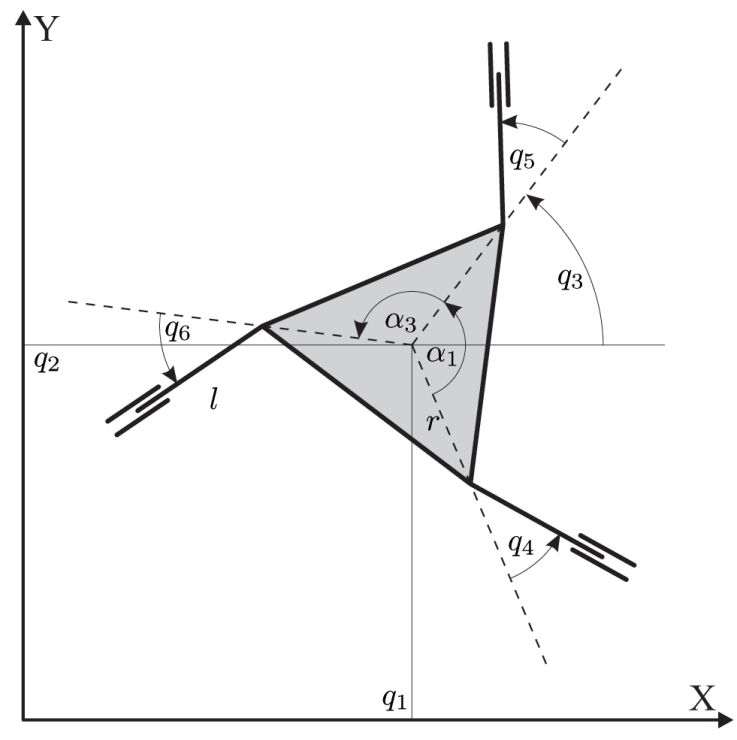

Figure 1: Trident snake

vector of generalized coordinates $q=\left(x, y, \theta, \phi_{1}, \phi_{2}, \phi_{3}\right)^{T} \in \mathbf{R}^{6}$ describes the position and orientation of the robot's body, and the angular position of each link. The links have the same length $l$, the body triangle is equilateral, with radius of the circumscribed circle equal to $r$. 
The kinematics of the trident snake mobile robot are represented by the driftless control system of the form (2),

$$
\dot{q}=G(q) u, \quad k(q)=\left(q_{1}, q_{2}, q_{3}\right)^{T},
$$

steered by three controls $u=\left(u_{1}, u_{2}, u_{3}\right)^{T} \in \mathbf{R}^{3}$. We shall solve the motion planning problem for two different representations of the robot's kinematics, see [14]: the first, when the controls are related to the position and orientation of the body (position and orientation control), and the second, when the controls are velocities of the joints (joint angle control).

In the position and orientation control case the control matrix $G(q)$ is as follows [5]

$$
G(q)=\left[\begin{array}{ccc}
\cos \theta & -\sin \theta & 0 \\
\sin \theta & \cos \theta & 0 \\
0 & 0 & 1 \\
\frac{1}{l} \sin \left(\phi_{1}+\alpha_{1}\right) & -\frac{1}{l} \cos \left(\phi_{1}+\alpha_{1}\right) & -\frac{1}{l}\left(l+r \cos \phi_{1}\right) \\
\frac{1}{l} \sin \left(\phi_{2}+\alpha_{2}\right) & -\frac{1}{l} \cos \left(\phi_{2}+\alpha_{2}\right) & -\frac{1}{l}\left(l+r \cos \phi_{2}\right) \\
\frac{1}{l} \sin \left(\phi_{3}+\alpha_{3}\right) & -\frac{1}{l} \cos \left(\phi_{3}+\alpha_{3}\right) & -\frac{1}{l}\left(l+r \cos \phi_{3}\right)
\end{array}\right]=\left[\begin{array}{l}
G_{1}(\theta) \\
G_{2}(\phi)
\end{array}\right] .
$$

The trident snake kinematics subject to the joint angle control are obtained by employing a feedback $v=G_{2}(\phi) u$. Therefore, the kinematics representation for the joint angle control case takes the form (42), with the control matrix [14]

$$
G(q)=\left[\begin{array}{c}
-\frac{1}{a}\left[\begin{array}{lll}
f_{1} & f_{2} & f_{3} \\
g_{1} & g_{2} & g_{3} \\
h_{1} & h_{2} & h_{3}
\end{array}\right] \\
I_{3}
\end{array}\right]
$$

where

$$
\begin{aligned}
a & =\frac{\left(l+r \cos \phi_{1}\right) \sin \left(\phi_{3}-\phi_{2}+\frac{2 \pi}{3}\right)+\left(l+r \cos \phi_{2}\right) \sin \left(\phi_{1}-\phi_{3}+\frac{2 \pi}{3}\right)+\left(l+r \cos \phi_{3}\right) \sin \left(\phi_{2}-\phi_{1}+\frac{2 \pi}{3}\right)}{l^{3}}, \\
f_{i} & =\frac{\left(l+r \cos \phi_{i+2}\right) \cos \left(\phi_{i+1}+\alpha_{i+1}+\theta\right)-\left(l+r \cos \phi_{i+1}\right) \cos \left(\phi_{i+2}+\alpha_{i+2}+\theta\right)}{l^{2}}, \\
g_{i} & =\frac{\left(l+r \cos \phi_{i+2}\right) \sin \left(\phi_{i+1}+\alpha_{i+1}+\theta\right)-\left(l+r \cos \phi_{i+1}\right) \sin \left(\phi_{i+2}+\alpha_{i+2}+\theta\right)}{l^{2}}, \\
h_{i} & =-l^{-2} \sin \left(\phi_{i+1}-\phi_{i+2}+\alpha_{i+1}-\alpha_{i+2}\right),
\end{aligned}
$$

with indexes $i$ to be counted modulo 3 (i.e. $\alpha_{4}=\alpha_{1}$,). In both cases, the following values of geometric parameters are assumed: $l=0.12 m, r=0.12 m$, and $\alpha_{1}=-\frac{2 \pi}{3}, \alpha_{2}=0$, $\alpha_{3}=\frac{2 \pi}{3}$.

In order to compute DCJI we need also the dynamics equations of the trident snake. To this aim we shall exploit the results of [15]. According to this reference, the entries 
of the inertia matrix of the trident snake $M(q)=M^{T}(q)=\left[m_{i j}\right], i, j=1, \ldots, 6$ are equal to

$$
\begin{aligned}
& m_{11}=m_{22}=m_{c}+3 m_{l}+3 m_{m}, \quad m_{14}=-l\left(m_{w}+\frac{m_{l}}{2}\right) \sin \left(\alpha_{1}+\phi_{1}+\theta\right), \\
& m_{15}=-l\left(m_{w}+\frac{m_{l}}{2}\right) \sin \left(\alpha_{2}+\phi_{2}+\theta\right), m_{16}=-l\left(m_{w}+\frac{m_{l}}{2}\right) \sin \left(\alpha_{3}+\phi_{3}+\theta\right), \\
& m_{24}=l\left(m_{w}+\frac{m_{l}}{2}\right) \cos \left(\alpha_{1}+\phi_{1}+\theta\right), \quad m_{25}=l\left(m_{w}+\frac{m_{l}}{2}\right) \cos \left(\alpha_{2}+\phi_{2}+\theta\right), \\
& m_{26}=l\left(m_{w}+\frac{m_{l}}{2}\right) \cos \left(\alpha_{3}+\phi_{3}+\theta\right), \\
& m_{34}=I_{0 w}+m_{w} l\left(l+r \cos \left(\phi_{1}\right)\right)+\frac{1}{6} m_{l} l\left(2 l+3 r \cos \left(\phi_{1}\right)\right) \\
& m_{35}=I_{0 w}+m_{w} l\left(l+r \cos \left(\phi_{2}\right)\right)+\frac{1}{6} m_{l} l\left(2 l+3 r \cos \left(\phi_{2}\right)\right) \\
& m_{36}=I_{0 w}+m_{w} l\left(l+r \cos \left(\phi_{3}\right)\right)+\frac{1}{6} m_{l} l\left(2 l+3 r \cos \left(\phi_{3}\right)\right) \\
& m_{44}=m_{55}=m_{66}=I_{0 w}+m_{w} l^{2}+\frac{1}{3} m_{l} l^{2}, \\
& m_{13}=-m_{w} l\left(\sin \left(\alpha_{1}+\phi_{1}+\theta\right)+\sin \left(\alpha_{2}+\phi_{2}+\theta\right)+\sin \left(\alpha_{3}+\phi_{3}+\theta\right)\right) \\
& \quad-m_{w} r\left(\sin \left(\alpha_{1}+\theta\right)+\sin \left(\alpha_{2}+\theta\right)+\sin \left(\alpha_{3}+\theta\right)\right)-\frac{1}{2} m_{l}\left(2 r \left(\sin \left(\alpha_{1}+\theta\right)\right.\right. \\
& \left.\quad+\sin \left(\alpha_{2}+\theta\right)+\sin \left(\alpha_{3}+\theta\right)\right)+l\left(\sin \left(\alpha_{1}+\phi_{1}+\theta\right)+\sin \left(\alpha_{2}+\phi_{2}+\theta\right)\right. \\
& \left.\left.\quad+\sin \left(\alpha_{3}+\phi_{3}+\theta\right)\right)\right), \\
& \left.\left.\quad+\cos \left(\alpha_{3}+\phi_{3}+\theta\right)\right)\right)+m_{m} r\left(\cos \left(\theta+\alpha_{1}\right)+\cos \left(\theta+\alpha_{2}\right)+\cos \left(\theta+\alpha_{3}\right)\right), \\
& \quad+m_{w} r\left(\cos \left(\alpha_{1}+\theta\right)+\cos \left(\alpha_{2}+\theta\right)+\cos \left(\alpha_{3}+\theta\right)\right)+\frac{1}{2} m_{l}\left(2 r \left(\cos \left(\alpha_{1}+\theta\right)\right.\right. \\
& \left.\quad+\cos \left(\alpha_{2}+\theta\right)+\cos \left(\alpha_{3}+\theta\right)\right)+l\left(\cos \left(\alpha_{1}+\phi_{1}+\theta\right)+\cos \left(\alpha_{2}+\phi_{2}+\theta\right)\right. \\
& m_{33}=I_{0}+3 I_{0 w}+3 m_{w}\left(r^{2}+l^{2}\right)+2 m_{w} r l\left(\cos \left(\phi_{1}\right)+\cos \left(\phi_{2}\right)+\cos \left(\phi_{3}\right)\right)+ \\
& m_{l}\left(l^{2}+3 r^{2}+l r\left(\cos \left(\phi_{1}\right)+\cos \left(\phi_{2}\right)+\cos \left(\phi_{3}\right)\right)\right)+6 m_{m} r^{2} . \\
& \\
& \left.\left.\quad \alpha_{1}+\phi_{1}+\theta\right)+\cos \left(\alpha_{2}+\phi_{2}+\theta\right)+\cos \left(\alpha_{3}+\phi_{3}+\theta\right)\right)
\end{aligned}
$$

Parameters of the robot's dynamics are the following: the body mass $m_{0}=0.52 \mathrm{~kg}$, the wheel mass $m_{w}=0.03 \mathrm{~kg}$, the wheel radius $r_{w}=0.02 \mathrm{~m}$, the wheel width $d=0.01 \mathrm{~m}$, the link mass $m_{l}=0.07 \mathrm{~kg}$, the motor mass $m_{m}=0.055 \mathrm{~kg}$, the total mass $m_{c}=m_{0}+3\left(m_{w}+\right.$ $\left.m_{m}+m_{l}\right)$, and the moments of inertia $I_{0}=\frac{m_{0} r^{2}}{4}, I_{0 w}=\frac{m_{w}\left(3 r_{w}^{2}+d^{2}\right)}{12}$.

Now, we shall solve the motion planning problem for the trident snake kinematics defined by (42) with substitution for the control matrix of either (43) or (44). The parametric motion planning algorithm (41) will be employed. The motion planning problem consists in driving the system (42) from $q_{0}=(0,0,0,0,0)$ to the desired $y_{d}=(0.5,0.5, \pi)$, over the time horizon $T=2$. All control functions have been chosen in the form of the trigonometric series including a constant term and 2 harmonics; this gives the parametric endogenous configuration space of dimension $s=15$. 

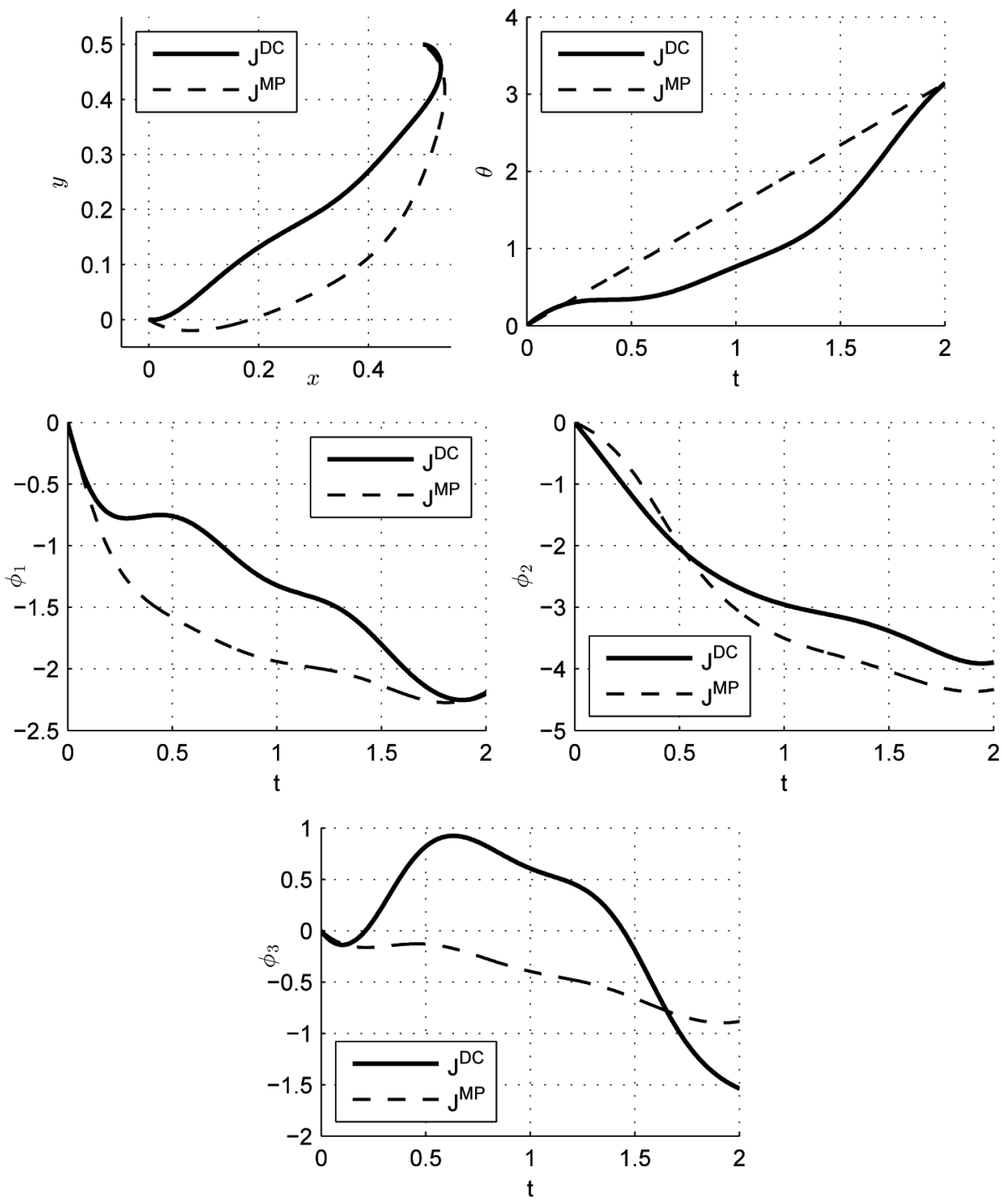

Figure 2: Plan of motion - the position and orientation control

Figures 2 and 3 show the solutions of the motion planning problem obtained by means of the DCJI and, for the comparison, using the Jacobian pseudoinverse, both in the parametric version. The differences between the solutions are exposed in Tabs. 1 and 2 , using two numerical parameters. The parameter named curve length expresses the length of the path of the body in XY plane. The second parameter, called area, denotes the area of the region determined by the XY path and the line connecting the initial point and the desired location. These two parameters could be treated as indicators of how 

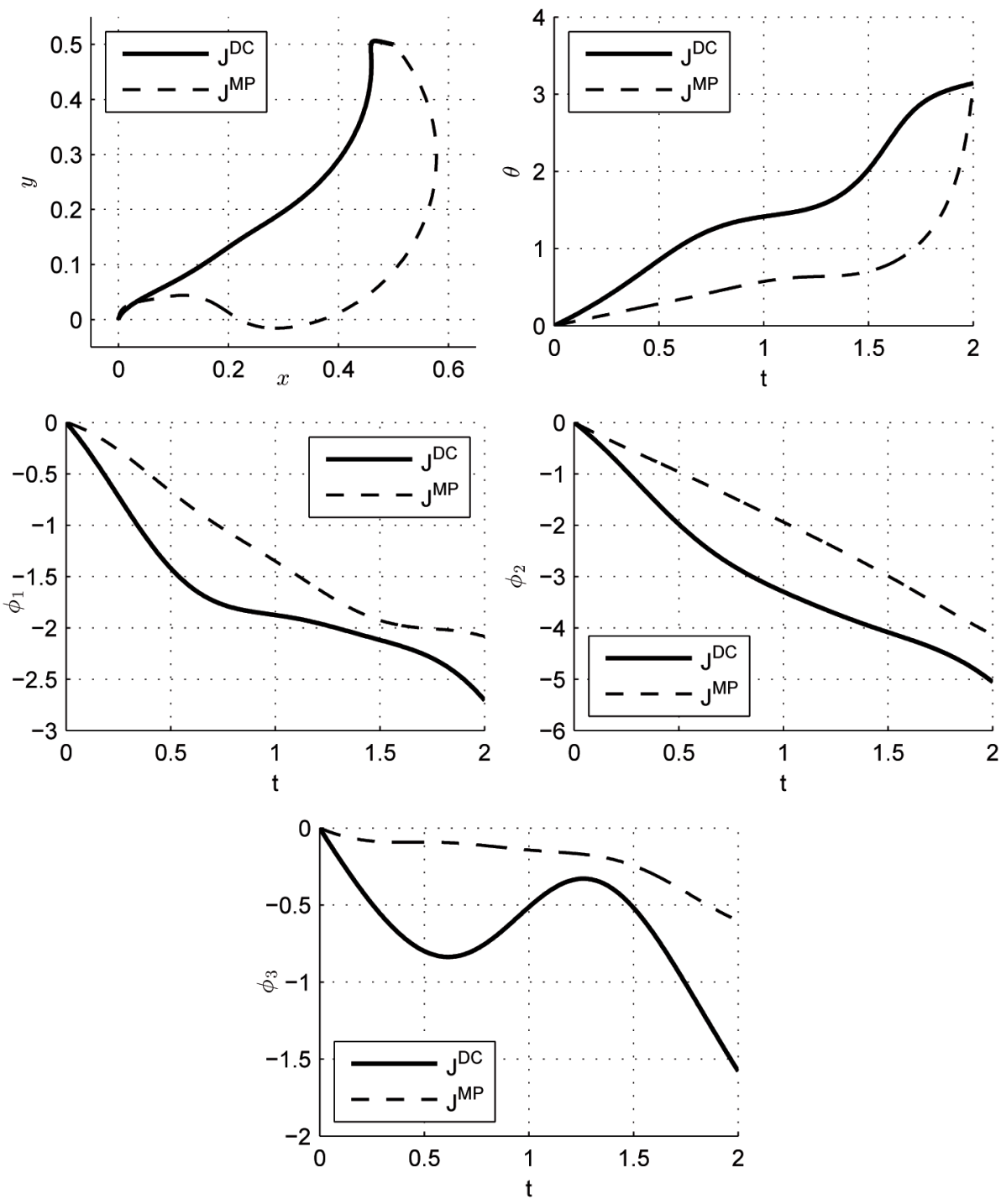

Figure 3: Plan of motion - the joint angle control

the solution differs from the shortest one in the plane (in the Euclidean metric). In this context it is worth to mention that for the best possible solution the curve length is equal $\sqrt{2} / 2 \approx 0.707$. One can observe that in both cases the solution provided by the dynamically consistent Jacobian inverse is only about by $7-8 \%$ longer than the segment of the straight line. The analysis of the Moore-Penrose Jacobian inverse shows that this difference rises to about $23 \%$ in position and orientation control case and exceeds $40 \%$ when the joint angles are controlled. This fact can be also observed when the area parameter is 
examined. When the position and orientation of the trident snake is controlled the area for the Jacobian pseudoinverse solution is twice as large than for DCJI, and this ratio grows even larger in the second case. The time required for the calculation depends on the complexity of the system's equations. One iteration of the dynamically consistent Jacobian algorithm takes about $0.1 \mathrm{~s}$ for the position and orientation control and $1.9 \mathrm{~s}$ for the joint angle control, and respectively $0.05 \mathrm{~s}$ and $1.6 \mathrm{~s}$ when we apply the Jacobian pseudoinverse algorithm ( $\mathrm{PC}$ with $3.40 \mathrm{GHz}$ processor). These times are not critical, as the motion planning may be performed off-line.

Table 1: Length and area - the position and orientation control

\begin{tabular}{|c|c|c|}
\hline & $J^{M P}$ & $J^{D C}$ \\
\hline curve length & 0.872867 & 0.778952 \\
\hline area & 0.105765 & 0.0467873 \\
\hline
\end{tabular}

Table 2: Length and area - the joint angle control

\begin{tabular}{|c|c|c|}
\hline & $J^{M P}$ & $J^{D C}$ \\
\hline curve length & 1.01162 & 0.766437 \\
\hline area & 0.148061 & 0.0450832 \\
\hline
\end{tabular}

\section{Conclusion}

We have studied the dynamically consistent Jacobian inverse (DCJI) for nonholonomic robotic systems within the endogenous configuration space approach. The geometric method of deriving this inverse has been examined that relies on a Riemannian metric in the endogenous configuration space, and extracts the consistency from a commutative diagram of maps related to the Jacobian and the Riemannian metric. A motion planning algorithm has been designed involving DCJI. For computational purposes a parametric version of this algorithm has been provided. Two motion planning problems for the trident snake have been solved. Two performance indicators disclosed that DCJIbased motion planning outperforms that relying on the Jacobian pseudoinverse. More comprehensive performance evaluation of DCJI will be a subject of our future research. 


\section{References}

[1] A. Ben-IsRael and T.N.E. Greville: Generalized Inverses. Springer, 2003.

[2] S. Chiaverini, G. Oriolo and I.D. WALKer: Kinematically Redundant Maniulators. In: Springer Handbook of Robotics, B. Siciliano, O. Khatib (Eds), Springer, 2008, 245-265.

[3] E. Demircan, ET AL.: Human Motion Reconstruction and Synthesis of Human Skills. Advances in Robot Kinematics: Motion in Man and Machine, Springer, 2010, 283-292.

[4] E. Demircan, ET AL.: Muscle force transmission to operational space accelerations during elite golf swings. Proc. IEEE Int. Conf. on Robotics and Automation, (2012), 1464-1469.

[5] M. IsHIKAWA: Trident snake robot: Locomotion analysis and control. Proc. IFAC Symp. on Nonlinear Control Systems (NOLCOS), (2004), 1169-1174.

[6] M. Ishikawa, Y. Minati and T. Sugie: Development and control experiment of the trident snake robot. IEEE/ASME Trans. Mechatronics, 15 (2010), 9-15.

[7] K. KAZEROUNIAN and Z. WANG: Global versus local optimization in redundancy resolution of robotic manipulators. Int. J. Robotics Research, 7 (1988), 3-12.

[8] O. KнAтIB: Motion/force redundancy of manipulators. 1990 Japan-USA Symp. on Flexible Automation, (1990), 337-342.

[9] O. Kнатів: Inertial properties in robotics manipulation: An object-level framework. Int. J. Robotics Research, 14 (1995), 19-36.

[10] O. KhatiB, ET AL.: Whole-body dynamic behavior and control of human-like robots. Int. J. Humanoid Robotics, 1 (2004), 29-43.

[11] R. Montgomery: A Tour of Subriemannian Geometries, their Geodesics and Applications. AMS, Providence, Rhode Island, 2002.

[12] J. NAKANiShi, ET AL.: Operational space control: A theoretical and empirical comparison. Int. J. Robotics Research, 27 (2008), 737-757.

[13] J.P. OSTROWSKI, ET AL.: The mechanics of undulatory locomotion: The mixed kinematic and dynamic case. Proc. IEEE Int. Conf. on Robotics and Automation, (1995), 1-15.

[14] D. Paszuk, K. TChoń and Z. Pietrowska: Motion planning of the trident snake robot equipped with passive or active wheels. Bulletin of the Polish Academy of Sciences Technical Sciences, 60 (2012), 547-554. 
[15] Z. PieTROwSKA: Kinematics, dynamics, and control of a trident snake type nonholonomic system. Master's Thesis, Wocław University of Technology, 2012 (in Polish).

[16] Z. PieTrowsKa and K. TCHOŃ: Dynamics and motion planning of Trident Snake Robot. J. Intelligent and Robotic Systems, 75 (2014), 17-28.

[17] J. RATAJCZAK and K. TCHOŃ: Dynamically consistent Jacobian inverse for mobile manipulators. Int. J. Control, 89 (2015), 1-16.

[18] L. Sentis, J. Park and O. Khatib: Compliant control of multicontact and center-of-mass behaviors in humanoid robots. IEEE Trans. Robotics, 26 (2010), 483-501.

[19] E.D. SonTAG: Mathematical Control Theory. Springer-Verlag, New York, 1999.

[20] H.J. Sussmann: A continuation method for non-holonomic path finding problems. Proc. 32nd IEEE Control and Decision Conference, (1993), 2718-2723.

[21] K. TCHOŃ and J. JAKUBIAK: Endogenous configuration space approach to mobile manipulators: a derivation and performance assessment of Jacobian inverse kinematics algorithms. Int. J. Control, 76 (2003), 1387-1419.

[22] K. TChoŃ and J. RATAJCZAK: Dynamically consistent Jacobian inverse for nonholonomic robotic systems. Nonlinear Dynamics, 85 (2016), 107-122.

[23] K. TCHOŃ: Endogenous Configuration Space Approach: An Intersection of Robotics and Control Theory. In: Nonlinear Systems, N. van de Wouw et al. (Eds), Springer, 2017, 209-234. 